\title{
EVALUACIÓN DEL EFECTO DEL FOLLAJE DE ÁRBOLES FORRAJEROS Y OLIGOQUETOS EN EL CRECIMIENTO DEL SORGO EN CONDICIONES DE INVERNADERO
}

\author{
Marco Ávila, ${ }^{1}$ Francisco Bautista,,${ }^{1,2}$ Esperanza Huerta ${ }^{3}$ \& Virginia \\ MELÉNDEZ ${ }^{1}$ \\ ${ }^{1}$ Cuerpo Académico de Ecología Tropical, Campus de Ciencias Biológicas y Agropecuarias,
} Universidad Autónoma de Yucatán. km 15.5 carretera Mérida-Xmatkuil, CP. 97000, Mérida Yucatán, México.

${ }^{2}$ Centro de Investigaciones en Geografía Ambiental, UNAM. Antigua Carretera a Pátzcuaro No. 8701 Col. Ex-Hacienda de San José de La Huerta CP. 58190 Morelia Michoacán, México. leptosol@ciga. unam.mx

${ }^{3}$ Manejo y Fertilidad de Suelos. El Colegio de la Frontera Sur, Unidad Villahermosa, km 15.5 Carr. Villahermosa-Reforma, Ranchería Guineo 2a Sección. CP. 86280, Villahermosa Tabasco, México.

Ávila, M., F. Bautista, E. Huerta \& V. Meléndez. 2010. Evaluación del efecto del follaje de árboles forrajeros y oligoquetos en el crecimiento del sorgo en condiciones de invernadero. Acta Zoológica Mexicana (n.s.), Número Especial 2: 227-239.

RESUMEN. Bajo condiciones de invernadero se analizó el efecto de la calidad del follaje de las especies arbóreas Diospyros cuneata, Bursera simaruba, Caesalpinia gaumeri, Randia longiloba, Gymnopodium floribundum y Piscidia piscicula, y de la lombriz de tierra Dichogaster bolaui en el crecimiento del sorgo. Se usaron cubetas de plástico conteniendo $2 \mathrm{~kg}$ de suelo, $100 \mathrm{~g}$ de follaje de cada especie y 1.2 g de lombrices. El diseño utilizado fue completamente al azar y los datos se analizaron con análisis de varianza y con un análisis de componentes principales para conocer la relación entre la calidad del follaje, el crecimiento del sorgo y la biomasa de lombrices. El follaje de B. simaruba y G. floribundum que aportaron las mayores cantidades de fósforo favorecieron la mayor producción de materia seca del sorgo, con o sin lombrices, aunque las mayores alturas de esta planta se correlacionaron con contenidos altos de Fibra Detergente/N. La aplicación de lombrices ocasionó un aumento de la biomasa total y la altura de las plantas de sorgo. El follaje de R. longiloba y $C$. gaumeri favorece a las poblaciones de D. bolaui pero no el crecimiento del sorgo. En el grupo de tratamientos con lombrices, el follaje de $P$. piscicula y $D$. cuneata mantuvo poblaciones favorables de lombrices, pero no beneficiaron el crecimiento del sorgo.

Palabras clave: Lombrices de tierra, calidad del follaje, crecimiento vegetal.

Ávila, M., F. Bautista, E. Huerta \& V. Meléndez. 2010. Evaluation of the effect of fodder tree leaflitter and earthworms on sorghum growth in the greenhouse. Acta Zoológica Mexicana (n.s.), Número Especial 2: 227-239.

ABSTRACT. Under greenhouse conditions we analyzed the effect of litter quality of the arboreal species Diospyros cuneata, Bursera simaruba, Caesalpinia gaumeri, Randia longiloba, Gymnopodium

Recibido: 16/05/2008; aceptado: 08/01/2010. 
floribundum and Piscidia piscicula, and inoculation of the earthworm Dichogaster bolaui, on sorghum growth. Plastic pots were used containing $2 \mathrm{~kg}$ of soil, $100 \mathrm{~g}$ of foliage of each plant and $1.2 \mathrm{~g}$ of earthworms. The experimental design was completely randomized and the data submitted to ANOVA and principal components analysis to study the relationships between litter quality, sorghum growth and earthworm biomass. The foliage of B. simaruba and G. floribundum that have higher phosphorus favored the higher sorghum biomass, with or without earthworms, although plant heights were correlated with higher ratios of Detergent Fibre/N. Earthworm inoculation increased total biomass and the height of the sorghum plants. The foliage of $R$. longiloba and $C$. gaumeri favors the populations of $D$. bolaui but not sorghum plant growth. In the group of treatments with earthworms, the foliage of P. piscipula and D. cuneata supported favorable populations of earthworms, but did not benefit sorghum growth.

Key words: Earthworms, litter quality, plant growth.

\section{INTRODUCCIÓN}

En Yucatán México, un millón de ha del territorio que inicialmente contaba con vegetación de selva baja y selva mediana se encuentra ahora con pastizales; además existen 1.5 millones de ha perturbados (Flores \& Espejel 1994). Las actividades agrícolas y pecuarias extensivas de roza, tumba y quema son de escasa productividad, debido a condiciones ambientales de suelos someros y clima de trópico subhúmedo y semiárido (Bautista et al. 2005) y el alto grado de perturbación de los ecosistemas. En el mantenimiento de los pastizales se invierte gran cantidad de esfuerzo y recursos económicos en la fertilización, control de arvenses y el riego que sólo se resuelve temporalmente con su abandono y con la apertura de nuevas tierras para pastos.

Los sistemas silvopastoriles (SSP) son una opción de manejo agroforestal con objetivos pecuarios; si se establecen con base en la vegetación secundaria, es posible producir follaje durante la época seca, su establecimiento es económico y se favorece la conservación de diversos servicios ambientales que pueden coexistir con la ganadería tradicional, aunque para promoverlos se requiere mayor conocimiento técnico de su funcionamiento (Sosa et al. 2004).

Flores \& Bautista (2005) reportaron 211 especies forrajeras utilizadas por los mayas, entre las que destacan Diospyros cuneata, Bursera simaruba, Caesalpinia gaumeri, Randia longiloba, Gymnopodium floribundum y Piscidia piscicula. Estas plantas pueden ser utilizadas para el diseño de SSP previo reconocimiento, en un principio, de su calidad, el efecto del follaje en la fertilidad del suelo y el efecto del follaje en la fauna del suelo, incluyendo las poblaciones de lombrices de tierra.

Las lombrices de tierra modifican muchas de las propiedades de los suelos, las cuales afectan el crecimiento de las plantas y por lo tanto su producción (Brown et al. 1999). En particular, son bien conocidos los beneficios de las lombrices en la fertilidad de los suelos (Barley 1959, Syers \& Springett 1984, Edwards 2004, Huerta et al. 2007), especialmente la disponibilidad de N y P para las plantas.

Los factores considerados como más determinantes que afectan a la distribución $\mathrm{y}$ abundancia de poblaciones de lombrices son temperatura, humedad, calidad de 
la hojarasca, contenido de materia orgánica y pH (Satchell 1967, Shakir \& Dindal 1997, Lee 1985, Edwards \& Bohlen 1996, Cannavacciulo et al. 1998, Bhadauria et al. 2000). Se han realizado varios experimentos que demuestran que la hojarasca con altas concentraciones de substancias lignificadas y polifenoles son más difíciles de descomponer y son menos palatables para los oligoquetos. Su uso podría disminuir el desarrollo de las poblaciones de las lombrices de tierra y sus efectos benéficos sobre el suelo (Satchell 1967, Hendriksen 1990, Daniel 1991, Lavelle \& Gilot 1994, Ganihar 2003).

La calidad de la materia orgánica juega un papel relevante; lombrices como $\mathrm{Mi}$ Ilsonia anomala, Pontoscolex corethrurus, Balanteodrilus pearsei y Polypheretima elongata han incrementado su crecimiento bajo el efecto de materia orgánica fresca (Lavelle et al. 1989a, b, García \& Fragoso 2003, Huerta et al. 2005, Ortiz-Ceballos et al. 2005). Sin embargo, el comportamiento de las poblaciones de la especie Dichogaster bolaui Michaelsen y su respuesta a diversa calidad de la hojarasca no está bien entendida.

En la búsqueda de opciones de diseño de sistemas agroforestales en donde las especies forrajeras beneficien a la planta principal y a la comunidad de macroinvertebrados se llevó al cabo el presente trabajo, cuyo objetivo fue evaluar el efecto de la calidad de hojarasca de seis especies de árboles forrajeros en combinación con la lombriz de tierra D. bolaui sobre el crecimiento del sorgo (Sorghum vulgare).

\section{MATERIAL Y MÉTODOS}

El estudio se realizó en condiciones de invernadero utilizando material de la vegetación secundaria que proviene de una selva baja caducifolia de doce años de edad, ubicada en el rancho Saramuyo, municipio de Mérida, Yucatán, México. Se seleccionaron seis especies arbóreas consideradas forrajeras: D. cuneata (D), B. simaruba (B), C. gaumeri (C), R. longiloba (R), G. floribundum (G) y P. piscicula (P) (Flores \& Bautista 2005).

En Septiembre del 2004, se tomaron muestras de hojas y tallos menores de $1 \mathrm{~cm}$ de diámetro de las especies seleccionadas. Las muestras se secaron a $60^{\circ} \mathrm{C}$ durante $48 \mathrm{~h}$, en una estufa de aire forzado. Después se molieron con un molino eléctrico y se tamizaron a $2 \mathrm{~mm}$. Del material preparado se tomaron submuestras para analizar el contenido de: nitrógeno total (N) por el método de Kjeldahl (AOAC 1990), las concentraciones de fósforo por el método colorimétrico, compuestos fenólicos en una extracción de metanol acuoso al 50\% y Folin-Denis (Anderson \& Ingram 1993), fibra detergente (FD) ácida (FDA) y neutra (FDN) por el método de Van Soest et al. (1991).

Se colectó el suelo del horizonte superficial de un Leptosol hiperesquelético, el cual se tamizó a $2 \mathrm{~mm}$. El suelo presentó las siguientes propiedades fisicoquímicas: $0.61 \%$ de $\mathrm{N} ; 14.94 \%$ de materia orgánica; $46.31 \%$ de capacidad de campo; $27.55 \%$ de punto de marchitamiento permanente; $1.66 \mathrm{mg} \mathrm{kg}^{-1}$ de $\mathrm{P} ; 42.17 \mathrm{cmol} \mathrm{kg}^{-1}$ de ca- 
pacidad de intercambio catiónico; $1.12 \mathrm{cmol} \mathrm{kg}^{-1}$ de Na; $5.21 \mathrm{cmol} \mathrm{kg}^{-1}$ de $\mathrm{K} ; 46.55$ cmol kg-1 de Ca; $3.12 \mathrm{cmol} \mathrm{kg}^{-1}$ de $\mathrm{Mg}$, así como un $90 \%$ de pedregosidad y una distribución de partículas de $38 \%$ de arena, $32 \%$ de limo y $30 \%$ de arcilla.

La colecta de las lombrices se realizó manualmente de un lugar cercano a un corral de ovinos. La especie $D$. bolaui fue seleccionada para utilizarse en los experimentos por ser la más abundante en la región.

El experimento se estableció en invernadero, en un arreglo estadístico factorial $(7 \times 2$ niveles), con y sin residuos de las seis especies y con $(+\mathrm{L})$ y sin lombrices, con una distribución de los tratamientos completamente al azar y con 10 repeticiones.

Se prepararon 140 macetas utilizando $2 \mathrm{~kg}$ de suelo con $100 \mathrm{~g}$ de follaje seco de la especie indicada de acuerdo a los tratamientos. En los tratamientos donde se utilizaron lombrices, se colocaron $30 \mathrm{~g} \mathrm{~m}^{-2}$ de biomasa de lombriz de la especie $D$. bolaui (17-21 individuos) en cada maceta (1.2 g por maceta) de acuerdo a Brown et al. (1999) como significativo para producir efectos sobre las plantas.

El sorgo se sembró en las macetas el 29 de noviembre del 2004 y se mantuvieron bajo condiciones de invernadero por 90 días de cultivo. Se realizaron deshierbes mensuales. En la siembra se colocaron cinco semillas en cada maceta y se realizó un raleo en estado de plántula dejando sólo la planta más vigorosa a los 15 días. El cultivo se mantuvo a una humedad promedio de $70 \%$ de capacidad de campo, necesaria para el desarrollo de las lombrices y el cultivo. La temperatura del invernadero permaneció entre 23 y $30^{\circ} \mathrm{C}$.

El efecto de la calidad del follaje y las lombrices sobre las plantas de sorgo se evaluaron mediante la medición de la altura y peso seco (parte aérea, raíces y biomasa total) del sorgo a los 90 días de cultivo.

Se realizó un análisis de varianza de una vía para comparar todos los tratamientos a la vez; posteriormente se realizó un análisis de varianza de dos vías para verificar diferencias significativas entre los tratamientos (tipos de hojarasca y con y sin lombrices). El programa utilizado fue Statgraphics Plus versión 5.1 (1995).

Se realizó un análisis en componentes principales con 12 variables: las características del follaje ( $\mathrm{N}, \mathrm{P}$, porcentajes de fibra detergente), los parámetros del sorgo con y sin lombrices (altura, materia seca al final del experimento), y la biomasa de lombrices, con el programa ADE-4 (Thioulouse et al. 1997). Con el fin de observar que tan significativo era la disposición del tipo de hojarasca sobre el plano factorial se hizo también un test de Montecarlo.

\section{RESULTADOS}

\section{Composición química del follaje de los árboles forrajeros}

Los follajes que presentaron los mayores porcentajes de fibra detergente ácida (FDA), fibra detergente neutra (FDN) y proporción fibra detergente ácida-nitrógeno (FDA/ 
N) fueron B y G. El orden de los follajes de acuerdo a la proporción FDA/N fue: P > $\mathrm{D}>\mathrm{C}>\mathrm{R}>\mathrm{G}>\mathrm{B}$. Los contenidos más altos de fenoles estuvieron en el follaje de $\mathrm{G}$ y $\mathrm{C}$ y la menor concentración fue presentada por D. Para la relación fenoles - nitrógeno $(\mathrm{F} / \mathrm{N})$ fueron $\mathrm{G}$ y $\mathrm{C}$ las que obtuvieron las mayores proporciones y $\mathrm{D}, \mathrm{P}$ y $\mathrm{R}$ las menores (Cuadro I).

\section{Efecto del follaje y las lombrices en el crecimiento del sorgo}

Los mayores incrementos en la altura y producción de materia seca se obtuvieron con el testigo (T) y el tratamiento L (solo lombrices) (Fig. 1 y 2); sin embargo con estos tratamientos las plantas mostraron clorosis y presentaron desbalances nutrimentales, situación que no ocurrió con las plantas en los tratamientos de hojarasca.

Dentro de los tratamientos con follaje y lombrices, $\mathrm{G}+\mathrm{L}$ tuvo la mayor producción de materia seca aérea total, en parte aérea y en raíces $(7.8 \mathrm{~g}, \mathrm{~F}=9.40, \mathrm{P}<0.05$, Fig. 1 abc) y el mayor crecimiento ( $45.1 \mathrm{~cm}$; Fig. 2). Los tratamientos B + L, C + L, D $+\mathrm{L}, \mathrm{G}+\mathrm{L}, \mathrm{P}+\mathrm{L}$ y $\mathrm{R}+\mathrm{L}$ presentaron valores de altura del sorgo claramente mayores a sus respectivos tratamientos sin lombrices (Fig. 2). La aplicación de lombrices, por lo tanto, ocasionó un aumento significativo en la biomasa total y en la altura de las plantas de sorgo a los 90 días (Fig. 3).

Dentro de los tratamientos sin lombrices, para la altura $\mathrm{G}$ fomentó el mayor crecimiento y B la mayor materia seca aérea (Fig. 1c y 2). En general, la hojarasca de G y $\mathrm{B}$ presentaron los valores mayores en altura y biomasa total del sorgo a los 90 días de desarrollo en comparación con los otros tipos de hojarasca (Fig. 4).

\section{Efecto de la calidad del follaje en el crecimiento del sorgo y en las lombrices de tierra}

La hojarasca que aportó las mayores cantidades de fósforo favoreció la mayor producción de materia seca del sorgo, aunque las mayores alturas de esta planta se correlacionaron con contenidos altos de FD/N (Fig. 5a, círculo de correlación).

Cuadro I. Composición química e índices de calidad del follaje de las diferentes especies forrajeras de la vegetación secundaria (\%). FDA = Fibra detergente ácida; FDN = Fibra detergente neutra.

\begin{tabular}{lccccccc}
\hline \multicolumn{1}{c}{ Especie } & $\mathrm{P}$ & $\mathrm{N}$ & FDA & FDN & Fenoles & FDA/N & Fenoles/N \\
\hline G. floribundum & 0.30 & 1.74 & 39.74 & 56.89 & 3.13 & 22.83 & 1.70 \\
P. piscipula & 0.28 & 2.09 & 26.30 & 41.75 & 0.88 & 12.58 & 0.42 \\
R. longiloba & 0.28 & 1.81 & 27.62 & 47.07 & 1.25 & 15.25 & 0.69 \\
D. cuneata & 0.29 & 2.04 & 28.63 & 43.53 & 0.47 & 14.03 & 0.23 \\
C. gaumeri & 0.28 & 2.06 & 29.62 & 45.79 & 3.62 & 14.37 & 1.75 \\
B. simaruba & 0.33 & 1.88 & 46.62 & 56.59 & 1.90 & 24.79 & 1.01 \\
\hline
\end{tabular}



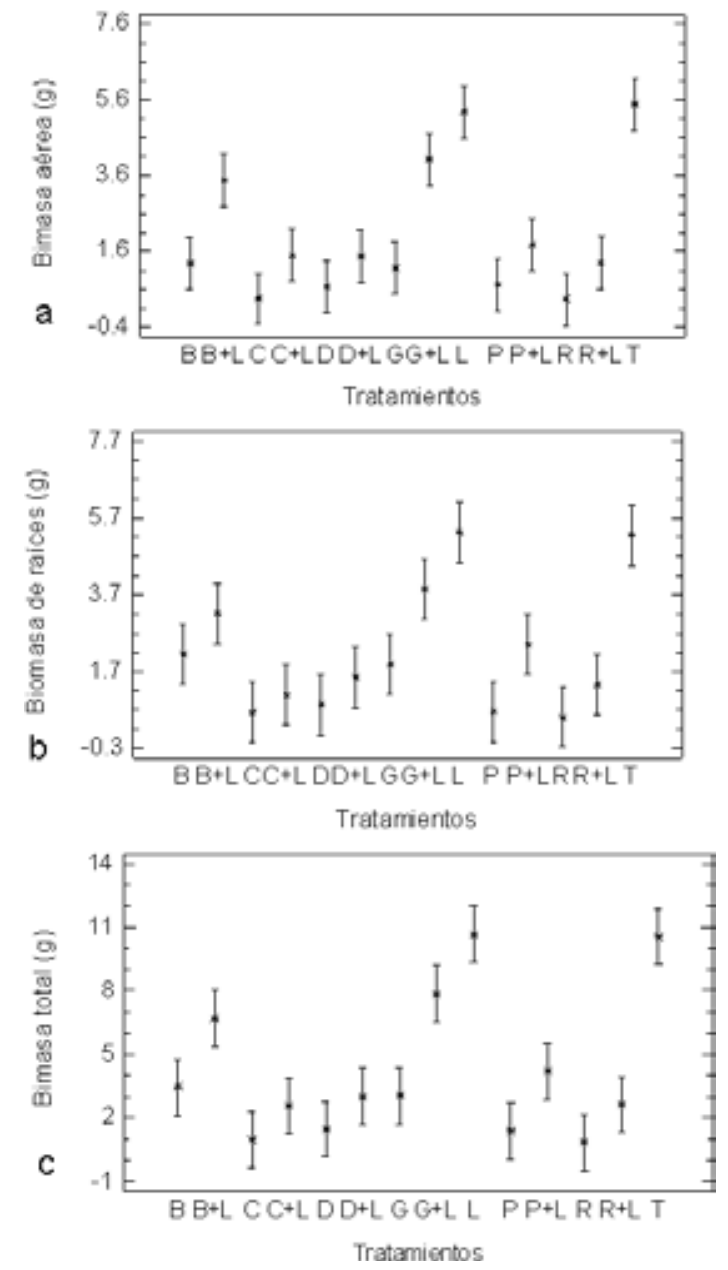

Figura 1. Efecto de la adición de hojarasca de D. cuneata (D), B. simaruba (B), C. gaumeri (C), R. longiloba (R), G. floribundum $(\mathrm{G})$ y P. piscicula $(\mathrm{P})$ y lombrices $(+\mathrm{L})$ en la biomasa del sorgo a los 90 días de crecimiento. En a, b y c con $\alpha=0.5$ y $\mathrm{P}<0.001$.

Los altos contenidos de $\mathrm{N}$ aportados por el follaje en los tratamientos con y sin lombrices no beneficiaron el crecimiento del sorgo: en el circulo de correlación obtenido con el análisis de componentes principales ( $87 \%$ de inercia en los primeros dos ejes), se observa claramente como el nitrógeno total se separó de la biomasa y altura del sorgo, y estas (altura y biomasa del sorgo) estuvieron correlacionadas con el tipo de fibras de las hojarascas. Las especies D, C y P a pesar de aportar las mayores cantidades de $\mathrm{N}$, no favorecieron al cultivo, mientras que $\mathrm{B}$ y $\mathrm{G}$ con bajos contenidos de este nutrimento, presentaron las mayores producciones de materia seca (Cuadro I y Fig. 1). 


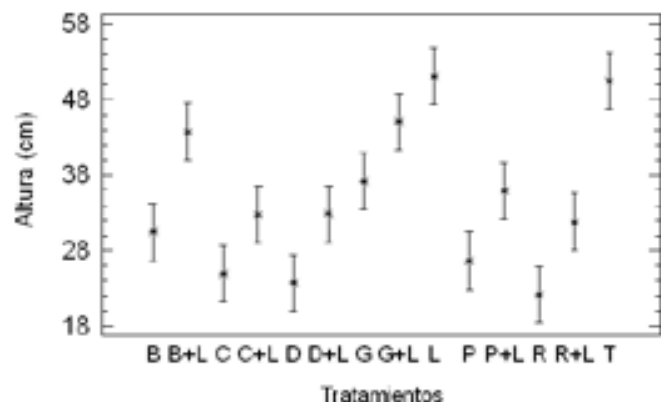

Figura 2. Efecto de la adición de hojarasca de D. cuneata (D), B. simaruba (B), C. gaumeri (C), $R$. longiloba $(\mathrm{R}), G$. floribundum $(\mathrm{G})$ y P. piscicula $(\mathrm{P})$ y lombrices $(+\mathrm{L})$ en la altura del sorgo a los 90 días de crecimiento, con $\alpha=0.5$ y $\mathrm{P}<0.001$.
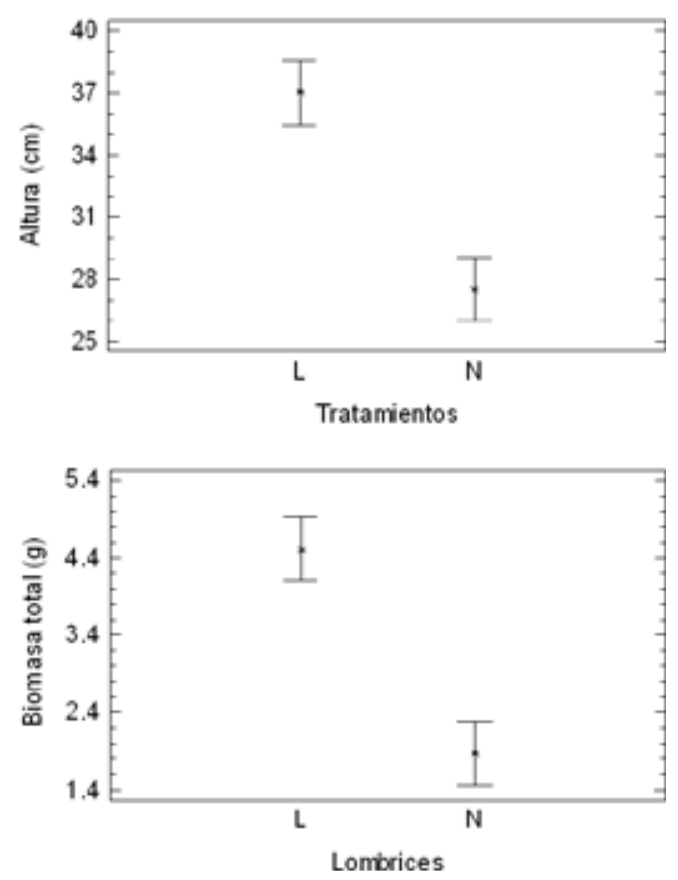

Figura 3. Efecto de las lombrices de tierra en la altura y biomasa total del sorgo a 90 días de desarrollo. Tratamientos con (L) y $\sin$ D. bolaui $(\mathrm{N})$. 
Ávila et al.: Efecto del follaje y lombrices en el sorgo
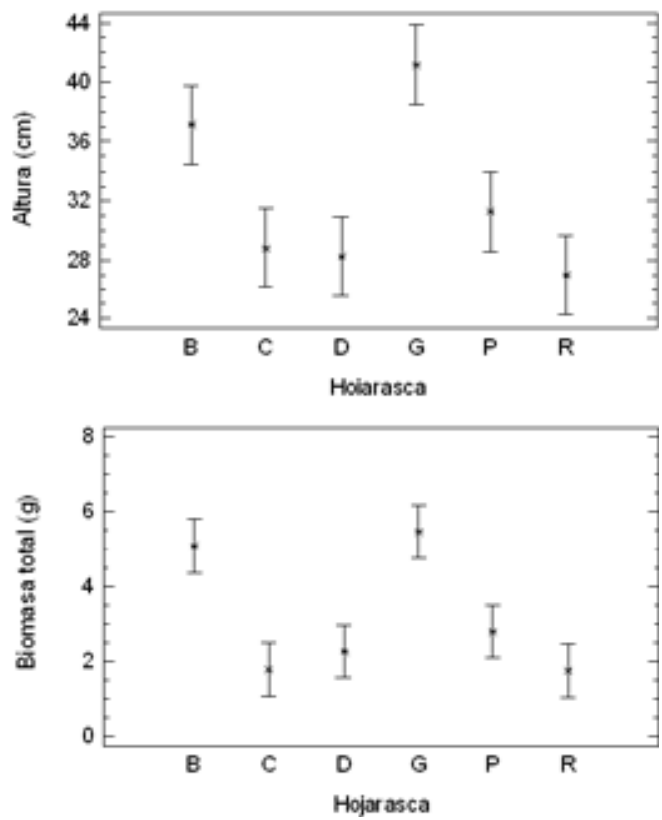

Figura 4. Efecto de la hojarasca de D. cuneata (D), B. simaruba (B), C. gaumeri (C), R. longiloba (R), G. floribundum $(\mathrm{G})$ y P. piscicula $(\mathrm{P})$ en la altura y biomasa del sorgo a los 90 días de desarrollo.
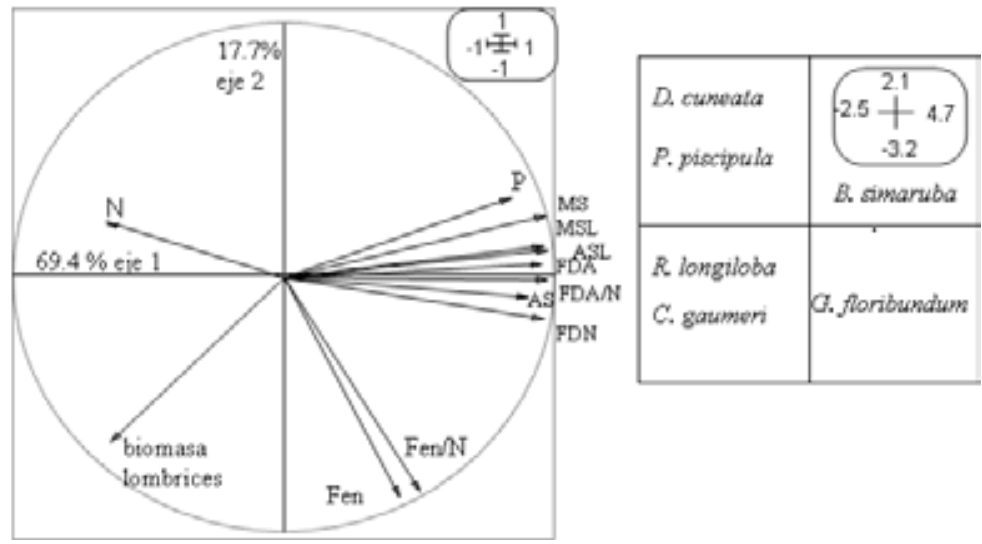

Figura 5. Representación en el plano factorial de las diferentes hojarascas después de un análisis en componentes principales (p:0.2) usando las variables del follaje $(\mathrm{N}=$ nitrógeno, $\mathrm{P}=$ Fósforo, FDA $=$ fibra detergente acida, FDN = Fibra detergente neutra, Fen = Fenoles) y los parámetros del sorgo con y $\sin$ lombrices $(\mathrm{AS}=$ altura del sorgo sin lombrices, $\mathrm{ASL}=$ Altura del sorgo con lombrices, $\mathrm{MS}=$ materia seca del sorgo sin lombrices, $\mathrm{MSL}=$ materia seca del sorgo con lombrices y $\mathrm{L}=$ biomasa de lombrices) 
El incremento de la biomasa de individuos de D. bolaui (lombriz epigea) fue independiente del crecimiento del sorgo: en el circulo de correlación se observa como se separó la biomasa de esta lombriz de todos los demás parámetros (Fig. 5a).

Los compuestos polifenólicos no mostraron un efecto inhibitorio en el crecimiento de las poblaciones de las lombrices y los follajes de $\mathrm{C}$ y $\mathrm{R}$ promovieron un aumento en la densidad y biomasa de las lombrices (Fig. 6). Las lombrices aplicadas en el tratamiento L se murieron y otras escaparon por falta de recursos alimenticios.
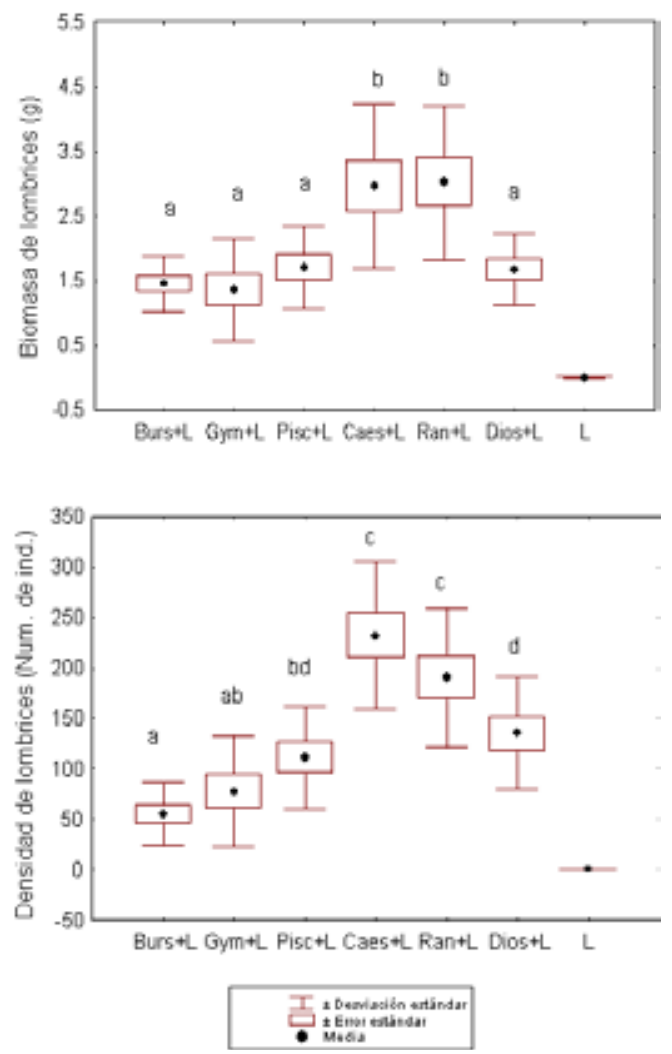

Figura 6. Efecto de la adición de D. cuneata (Dios), B. simaruba (Burs), C. gaumeri (Caes), $R$. longiloba (Ran), G. floribundum (Gym) y P. piscicula (Pisc) en la densidad y biomasa de lombrices (g por maceta). Letras iguales no son significativamente diferentes $(\mathrm{P}<0.05)$.

\section{DISCUSIÓN}

\section{Composición química del follaje de árboles forrajeros}

En los estudios de calidad de hojarasca se ha reconocido que la variación de las condiciones ambientales del sitio puede tener un efecto considerable en la composición 
química de la hojarasca generada por diferentes individuos de la misma especie de árboles (Berg \& Tamm 1991). Por lo tanto, la composición química de los árboles depende de muchos factores tales como la especie, parte de la planta, la proporción de hojas y tallos, edad del tejido, frecuencia de poda, clima y suelo (Palm 1995). En este estudio se utilizó follaje fresco que difiere de la hojarasca en que no ha traslocado sus nutrimentos. Por esta razón, se considera con un mayor valor nutrimental (Constantinides \& Fownes 1994).

\section{Efectos del follaje y de las lombrices de tierra en el crecimiento del sorgo}

Las lombrices usadas (D. bolaui) beneficiaron el crecimiento del cultivo de sorgo. Sin embargo, el efecto varió según el tipo de follaje usado. Hartemink \& O'Sullivan (2001) sugieren que los diversos tipos de follaje presentan diferentes patrones de descomposición y mineralización que afectan la fertilidad en diferentes niveles. Varios estudios realizados por Lavelle et al. (1994) muestran que la calidad de la materia orgánica aportada al suelo, determina las tasas de crecimiento de las poblaciones de oligoquetos, que repercuten en la fertilidad del suelo. Cheshire \& Griffits (1989) sugieren que la actividad de estos organismos acelera la liberación de los nutrimentos de residuos vegetales, pero el efecto en las plantas depende de la composición química del material orgánico aplicado y de las propiedades del suelo.

En este estudio las lombrices beneficiaron al cultivo cuando se combinaron con follaje de G, B y P, en comparación con los tratamientos de solo follaje. Entre los follajes aplicados con lombrices, los mejores resultados se obtuvieron con $\mathrm{G}$ y $\mathrm{B}$, tal vez por los mayores contenidos de fósforo y la dinámica de la mineralización del follaje.

Por ser de origen cárstico, los suelos del estudio contienen altos contenidos de calcio intercambiable ( $46.55 \mathrm{cmol} \mathrm{kg}^{-1} \mathrm{de} \mathrm{Ca}$ ) que puede formar uniones covalentes con los escasos fosfatos disponibles (1.66 $\mathrm{mg} \mathrm{kg}^{-1} \mathrm{de} \mathrm{P}$ ), limitando con esto la absorción de fósforo por las plantas (Weisbach et al. 2002, Bautista et al. 2003, 2005). Es posible que el mayor contenido de fósforo en el follaje de $\mathrm{G}$ y B, así como su menor calidad reflejada por los mayores valores de FDA/N ocasionen una descomposición más lenta, pero en sincronía con las necesidades nutrimentales del sorgo (Bautista et al. 2008).

Es importante recalcar que los datos se tomaron a los tres meses, después de establecerse el cultivo (inicio de la floración) y no se esperó hasta la cosecha. Brown et al. (1999) indican que los beneficios de las lombrices se pueden observar muchas veces en el rendimiento de la producción; sin embargo, el efecto benéfico de las lombrices en el crecimiento del sorgo en este estudio está claro y la mayor biomasa vegetal probablemente se reflejaría en una mayor producción de granos.

\section{Efecto del follaje en el crecimiento de las lombrices}

Los resultados del presente estudio muestran el efecto desfavorable del incremento de la proporción FDA/N en la densidad de los oligoquetos. Estudios realizados por 
Tian et al. (2000) sugieren que las especies como Chromolena odorata que producen hojarasca con contenidos bajos de polifenoles y altas concentraciones de nitrógeno favorecen las poblaciones de lombrices debido a que los recursos con un mejor grado de palatabilidad hacen que su descomposición sea mas rápida. En este estudio el follaje de los árboles forrajeros con altos contenidos de compuestos solubles y bajas concentraciones de fibras lignificadas como $\mathrm{R}$ y $\mathrm{C}$ favoreció la población de lombrices pero no al crecimiento de los cultivos.

En este estudio se encontró también que existen especies arbóreas como $\mathrm{R}$ y $\mathrm{C}$ que pueden beneficiar a la fauna edáfica, en este caso a las lombrices, de manera inmediata al liberar nutrimentos (bajos valores de FDA/N) con más facilidad, ayudando a iniciar el ciclo de descomposición de la materia orgánica y mineralización de nutrimentos, tal como lo señala y recomienda Ganihar (2003). De esta manera, al diseñar los sistemas agroforestales es recomendable la combinación de especies arbóreas para fomentar la diversidad en la calidad de los sustratos para que los sistemas sean estables ecológicamente (Swift 1985, Khanna, 1998).

El presente estudio encontró que de las seis especies evaluadas, la aplicación del follaje de dos de ellas (B. simaruba y G. floribundum) sin lombrices y tres de ellas ( $G$. floribundum, B. simaruba y $P$. piscipula) con lombrices favorecieron el crecimiento del sorgo. G. floribundum y B. simaruba presentaron altos contenidos de material fibroso y fósforo, que favorecieron el crecimiento del cultivo. Los árboles forrajeros que aportan follaje con bajas concentraciones de fibras lignificadas como $R$. longiloba y $C$. gaumeri favorecieron a las poblaciones de $D$. bolaui y no al crecimiento del sorgo. En el grupo de tratamientos con lombrices, el follaje de $P$. piscipula y D. cuneata mantuvo poblaciones favorables de lombrices, pero no beneficiaron a las plantas.

\section{AGRADECIMIENTOS}

A los fondos mixtos Yucatán proyecto YUC-2003-C0-054 y al Consejo Nacional de Ciencia y Tecnología por la beca a MA. A Hilda Rivas por el apoyo técnico. A dos revisores anónimos por sus valiosos comentarios que permitieron modificar y mejorar profundamente el artículo.

\section{LITERATURA CITADA}

Anderson, J. M. \& J. I Ingram. 1993. Tropical soil biology and fertility. A handbook of methods. CAB International. Wallingford.

AOAC 1990. Official methods of analysis. Association of Official Analytical Chemistry, Washington DC.

Bautista, F., J. Jiménez-Osornio, J. Navarro-Alberto, A. Manu \& R. Lozano 2003. Microrelieve y color del suelo como propiedades de diagnóstico en Leptosoles cársticos. Terra. 21: 1-11.

Bautista F., J. García \& A. Mizrahi 2005. Diagnóstico campesino de la situación agrícola en Hocabá, Yucatán. Terra Latinoamericana. 23(4): 571-580. 
Bautista F., C. Delgado \& H. Estrada 2008. Effect of legume mulches and cover crops on earthworms and snails. Tropical and Subtropical Agroecosystems. 8: 45-60.

Barley, K. P. 1959. Earthworms and soil fertility IV. The influence of earthworms on the physical properties of a red-brown earth. Australian Journal of Agricultural Research. 10: 371-376.

Berg, B. \& C. O. Tamm. 1991. Decomposition and nutrient dynamics of litter in long-term optimum nutrition experiments. Scandinavian Journal of Forest Research. 6: 305-321.

Bhadauria, T., P. S. Ramakrishnan \& K. N. Srivastava. 2000. Diversity and distribution of endemic and exotic earthworms in natural and regenerating ecosystems in the central Himalayas, India. Soil Biology and Biochemistry. 32: 2045-2054.

Brown, G., B. Pashanasi, C. Villenave, J. Patron, B. Senapati, S. Giri, I. Barois, P. Lavelle, E. Blanchart, R. Blakemore, A. Spain \& J. Boyer. 1999. Effects of Earthworms on plant production in tropics. Pp. 87-147. In: P. Lavelle, L. Brussaard and P. F. Hendrix (Eds.). Earthworm management in tropical agroecosystems. CAB International, Wallingford.

Cannavacciuolo, M., A. Bellido, D. Cluzeau, C. Gascuel \& P. Trehen. 1998. A geostatistical approach to the study of earthworm distribution in grassland. Applied Soil Ecology. 9: 345-349.

Cheshire, M. V. \& B. S. Griffiths. 1989. The influence of earthworms and crane fly larvae on decomposition of uniformly ${ }^{14} \mathrm{C}$ labeled plant material in soil. Soil Science. 40: 117-124.

Constantinides, M. \& J. H. Fownes. 1994. Nitrogen mineralization from leaves and litter of tropical plants: relationships to nitrogen, lignin and soluble polyphenol concentrations. Soil Biology and Biochemistry. 26: 49-55.

Daniel, O. 1991. Leaf litter consumption and assimilation by juveniles of Lumbricus terrestris L. (Oligochaeta, Lumbricidae) under different environment condition. Biology and Fertility of Soils. 12: 202-208.

Edwards, C. A. \& P. J. Bohlen. 1996. Biology and ecology of earthworms, $3^{\text {rd }}$ Ed. Chapman and Hall, London.

Edwards, C. A. 2004. The importance of earthworms as key representatives of soil fauna. Pp. 3-12. In: C. A. Edwards (Ed.). Earthworm ecology, $2^{\text {nd }}$ Ed. St. Lucie Press, Boca Raton.

Flores, J. S. \& C. I. Espejel. 1994. Tipos de vegetación de la Península de Yucatán. Pp. 73-126. In: Etnoflora Yucatanense, Vol. 3. Universidad Autónoma de Yucatán. Mérida.

Flores, S. \& F. Bautista. 2005. Inventario de plantas forrajeras utilizadas por los mayas en los paisajes geomorfológicos de la península de Yucatán. Pp 209-219. In: F. Bautista and G. Palacio (Eds.). Caracterización y manejo de suelos en la Península de Yucatán. UACAM-UADY-INE, México, D.F.

Ganihar, S. 2003. Nutrient mineralization and leaf litter preference by the earthworm Pontoscolex coretrhrus on iron ore mine wastes. Restoration Ecology. 11: 475-482.

García, J. A. \& C. Fragoso. 2003. Influence of different food substrates on growth and reproduction of two tropical earthworm species (Pontoxcolex corethrurus and Amynthas corticis). Pedobiologia. 47: 24-32.

Hartemink, A. E. \& J. N. O'Sullivan. 2001. Leaf litter decomposition of Piper auducum, Gliricidia sepium and Imperata cylindrical in the humid lowlands of Papua New Guinea. Plant and Soil. 230: $115-124$.

Hendriksen, N. B. 1990. Leaf litter selection by detrivore and geophagous earthworms. Biology and Fertility of Soils. 13: 6-10.

Huerta, E., D. de la O. de Dios \& G. Nuncio. 2007. Incremento de la fertilidad de suelos mediante el uso de lombrices de tierra (Glossoscolecidae y Acanthodrilidae) y leguminosas (Arachis pintoi) en un suelo de traspatio. Ciencia Ergo Sum. 14(2): 1-4.

Huerta, E., C. Fragoso, I. Barois \& P. Lavelle. 2005. Enhancement of growth and reproduction of the tropical earthworm Polypheretima elongata (Megascolecidae) by addition of Zea mays and Mucuna pruriens var. utilis litter to the soil. European Journal of Soil Biology. 41: 45-53. 
Khanna, P. K. 1998. Nutrient cycling under mixed-species tree systems in Southeast Asia. Agroforestry Systems. 38: 99-120.

Lavelle, P. \& C. Gillot. 1994. Priming effects of microorganisms on microflora: a key process of soil function? Pp. 133-180. In: K. Ritz, J. Dighton and K. E. Giller. (Eds.). Beyond the biomass. Compositional and functional analysis of soil microbial communities. Willey, Chichester.

Lavelle, P., M. Dangerfield, C. Fragoso, V. Eschenbrenner, D. López-Hernández, B. Pashanasi \& L. Brussaard. 1994. The relationship between soil macrofauna and tropical soil fertility. Pp. 137169. In: P. L. Woomer and M. J. Swift (Eds.). The biological management of tropical soil fertility. Wiley-Sayce.

Lavelle, P., R. Schaefer \& Z. Zaidi. 1989a. Soil ingestion and growth in Millsonia anomala, a tropical earthworm, as influenced by the quality of the organic matter ingested. Pedobiologia. 33: 379-388.

Lavelle, P., I. Barois, A. Martin, Z. Zaidi \& R. Schaefer. 1989b. Management of earthworm populations in agro-ecosystems: a possible way to maintain soil quality? Pp. 109-122. In: L. Bergstrom and M. Clarholm. Kluwer (Eds.). Ecology of arable land: perspectives and challenges. Kluwer Academic Publishers, Dordrecht.

Lee, K. E. 1985. Earthworms: their ecology and relationships with soils and land use. Academic Press, Sydney.

Ortiz-Ceballos, A. I., C. Fragoso, M. Equihua \& G. G. Brown. 2005. Influence of food quality, soil moisture and the earthworm Pontoscolex corethrurus on growth and reproduction of the tropical earthworm Balanteodrilus pearsei. Pedobiologia. 49: 89-98.

Palm, C. A. 1995. Contribution of agroforestry trees to nutrient requirements in intercropped plants. Agroforestry Systems. 30: 105-124.

Satchell, J. E. 1967. Lumbricidae. Pp. 259-322. In: L. Burgess and F. Raw (Eds.). Soil Biology. Academic Press, London.

Shakir, S. \& D. Dindal. 1997. Density and biomass of earthworms in forest and herbaceous microecosystems in Central New York, North America. Soil Biology and Biochemistry. 29: 275-285.

Sosa, R. E., R. D. Pérez, R. L. Ortega \& B. G. Zapata. 2004. Evaluación del potencial forrajero de árboles y arbustos tropicales para la alimentación de ovinos. Técnica Pecuaria en México. 42(2): 129-144.

Statistical Graphics Corporation. 1995. Statgraphics Plus version 5.1.

Swift, M. J. 1985. Tropical Soil Biology and Fertility (TSBF). Planning for Research. Biology International. Special Issue No. 9.

Syers, J. K. \& J. A. Springett. 1984. Earthworms and soil fertility. Plant and Soil. 79: 1-3.

Tian, G., J. A. Olimah, G. O. Adeoye \& B. T. Kang. 2000. Regeneration of earthworm populations in a degraded soil by natural and planted fallow under humid tropical conditions. Soil Science Society of America Journal. 64: 222-228.

Van Soest, P. J., J. B. Robertson \& B. A. Lewis. 1991. Methods for dietary fibers, neutral detergent fiber and non-starch polysaccharides in relation to animal nutrition. Journal of Dairy Science. 74: 35-83.

Weisbach, C., H. Tiessen \& J. Jiménez. 2002. Soil fertility during shifting cultivation in the tropical karst soils of Yucatán. Agronomie. 2: 253-263.

Thioulouse, J., D. Chessel, S. Dolédec \& J.-P. Olivier. 1997. ADE-4: a multivariate analysis and graphical display software. Statistics and Computing. 7: 75-83. 